\title{
Crystal structure of $(3 \mathrm{aS}, 6 \mathrm{aS})-3-[(1 R, 2 S, 3 S)-1,3: 2,4$-di- $O$-ethylidene- 1,2,3,4- tetrahydroxy-1-butyl]-3a,6a-dihydrofuro[2,3-d]isoxazole, $\mathrm{C}_{13} \mathrm{H}_{17} \mathrm{NO}_{6}$
}

\author{
S. Henkel, T. Leibold and V. Jäger \\ Universität Stuttgart, Institut für Organische Chemie, Pfaffenwaldring 55, D-70569 Stuttgart, Germany
}

Received January 13, 1997, CSD-No. 402797

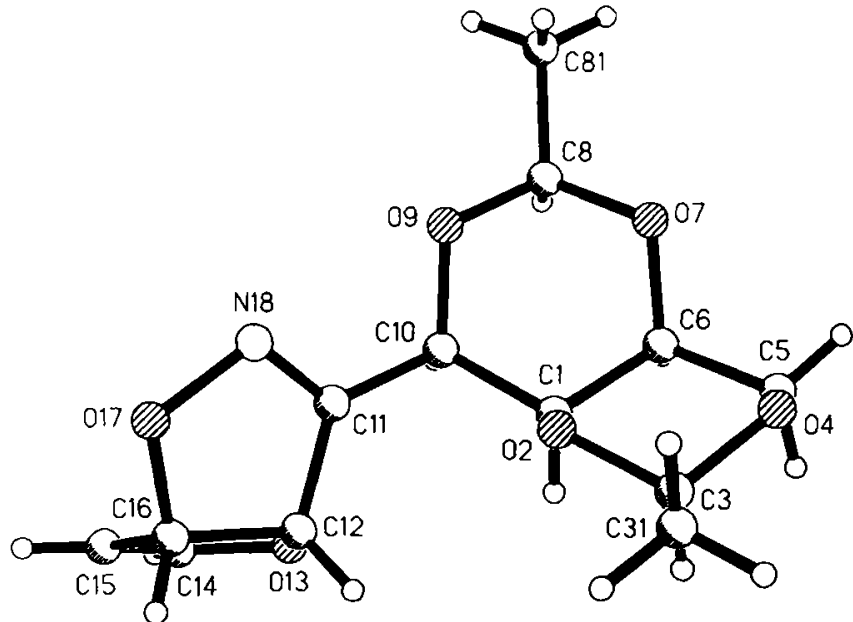

Source of material: The title compound was prepared (see ref. 1) by 1,3-dipolar cycloaddition of furan (see ref. 2 and 3 ) with the corresponding hydroximoyl chloride which was derived (see ref. 4) from the corresponding L-xylose oxime (see ref. 5). The mixture of diastereomeric cycloadducts $[(3 \mathrm{a} R, 6 \mathrm{a} R) /(3 \mathrm{aS}, 6 \mathrm{a} S)=70: 30]$ was separated by MPLC and both isomers were recrystallized from petroleum ether / ethyl acetate (see refs. 1 and 6).

$\mathrm{C}_{13} \mathrm{H}_{17} \mathrm{NO}_{6}$, orthorhombic, $P 2{ }_{2}{ }_{12}$ (No. 19), $a=6.541$ (1) $\AA$, $b=8.938(2) \AA, c=23.161(4) \AA, V=1354.1 \AA^{3}, Z=4, R(F)=0.046$, $R_{\mathrm{W}}\left(F^{2}\right)=0.112$.

Table 1. Parameters used for the $X$-ray data collection

Crystal:

Wavelength:

$\mu$ :

Diffractometer:

Scan mode:

$T_{\text {measurement: }}$

$2 \theta_{\max }:$

$N(h k h)_{\text {unique: }}$

Criterion for $I_{0}$ :

$N($ param) refined:

Programs: colorless block, size $0.4 \times 0.5 \times 0.75 \mathrm{~mm}$

Mo $K_{\alpha}$ radiation $(0.71073 \AA$ )

$1.11 \mathrm{~cm}^{-1}$

Nicolet P3

Wyckoff

$293 \mathrm{~K}$

$60^{\circ}$

2226

$I_{0}>2 \sigma\left(I_{0}\right)$

181

SHELXS-86, SHELXL-93

Table 2. Final atomic coordinates and displacement parameters (in $\AA^{2}$ )

\begin{tabular}{llllll}
\hline Atom & Site & \multicolumn{1}{l}{$y$} & $y$ & $U_{\text {iso }}$ \\
\hline H(1) & $4 a$ & $-0.1310(3)$ & $0.9820(2)$ & $0.86685(8)$ & 0.036 \\
H(3) & $4 a$ & $-0.2503(3)$ & $0.8097(3)$ & $0.9328(1)$ & 0.050 \\
H(31A) & $4 a$ & $-0.0322(5)$ & $0.7413(4)$ & $1.0086(1)$ & 0.095 \\
H(31B) & $4 a$ & $0.0390(5)$ & $0.6000(4)$ & $0.9740(1)$ & 0.095 \\
H(31C) & $4 a$ & $-0.1866(5)$ & $0.6110(4)$ & $0.9964(1)$ & 0.095 \\
H(5A) & $4 a$ & $-0.3983(3)$ & $0.7546(3)$ & $0.8437(1)$ & 0.053 \\
H(5B) & $4 a$ & $-0.3076(3)$ & $0.6241(3)$ & $0.8064(1)$ & 0.053 \\
H(6) & $4 a$ & $-0.1930(3)$ & $0.8709(2)$ & $0.77980(9)$ & 0.042 \\
H(8) & $4 a$ & $0.1165(4)$ & $0.8948(3)$ & $0.72995(9)$ & 0.049 \\
H(81A) & $4 a$ & $0.4342(5)$ & $0.7955(4)$ & $0.7045(1)$ & 0.090 \\
H(81B) & $4 a$ & $0.2625(5)$ & $0.6786(4)$ & $0.6905(1)$ & 0.090 \\
H(81C) & $4 a$ & $0.3981(5)$ & $0.6590(4)$ & $0.7458(1)$ & 0.090 \\
H(10) & $4 a$ & $0.1071(3)$ & $1.0683(2)$ & $0.80348(8)$ & 0.036 \\
H(12) & $4 a$ & $0.0695(4)$ & $1.1514(3)$ & $0.9385(1)$ & 0.061 \\
H(14) & $4 a$ & $0.2314(7)$ & $1.5140(4)$ & $0.8653(2)$ & 0.101 \\
H(15) & $4 a$ & $0.4939(6)$ & $1.4641(3)$ & $0.9313(2)$ & 0.087 \\
H(16) & $4 a$ & $0.3355(4)$ & $1.2485(3)$ & $0.9886(1)$ & 0.069 \\
\hline
\end{tabular}

Table 3. Final atomic coordinates and displacement parameters (in $\AA^{\mathbf{2}}$ )

\begin{tabular}{|c|c|c|c|c|c|c|c|c|c|c|}
\hline Atom & Site & $x$ & $y$ & $z$ & $U_{11}$ & $U_{22}$ & $U_{33}$ & $U_{12}$ & $U_{13}$ & $U_{23}$ \\
\hline$C(1)$ & $4 a$ & $-0.0283(3)$ & $0.9086(2)$ & $0.85465(8)$ & $0.0283(8)$ & $0.0276(8)$ & $0.0339(8)$ & $0.0033(8)$ & $-0.0018(7)$ & $0.0007(7)$ \\
\hline$O(2)$ & $4 a$ & $0.0341(2)$ & $0.8241(2)$ & $0.90368(6)$ & $0.0297(6)$ & $0.0393(7)$ & $0.0339(7)$ & $-0.0017(6)$ & $-0.0026(6)$ & $0.0089(6)$ \\
\hline $\mathrm{C}(3)$ & $4 a$ & $-0.1359(3)$ & $0.7415(3)$ & $0.9255(1)$ & $0.033(1)$ & $0.047(1)$ & $0.046(1)$ & $-0.001(1)$ & $0.0028(9)$ & $0.013(1)$ \\
\hline$C(31)$ & $4 a$ & $-0.0732(5)$ & $0.6666(4)$ & $0.9812(1)$ & $0.052(2)$ & $0.079(2)$ & $0.060(2)$ & $-0.003(2)$ & $0.000(1)$ & $0.037(2)$ \\
\hline$O(4)$ & $4 a$ & $-0.1957(3)$ & $0.6319(2)$ & $0.88547(8)$ & $0.0417(8)$ & $0.0376(8)$ & $0.066(1)$ & $-0.0076(7)$ & $-0.0035(8)$ & $0.0122(8)$ \\
\hline $\mathrm{C}(5)$ & $4 a$ & $-0.2737(3)$ & $0.7009(3)$ & $0.8344(1)$ & $0.0304(9)$ & $0.043(1)$ & $0.060(1)$ & $-0.0037(9)$ & $-0.008(1)$ & $-0.004(1)$ \\
\hline$C(6)$ & $4 a$ & $-0.1220(3)$ & $0.8080(2)$ & $0.80806(9)$ & $0.0329(9)$ & $0.0336(9)$ & $0.0386(9)$ & $0.0003(9)$ & $-0.0083(8)$ & $-0.0026(8)$ \\
\hline$O(7)$ & $4 a$ & $0.0341(3)$ & $0.7242(2)$ & $0.77893(7)$ & $0.0408(8)$ & $0.0371(7)$ & $0.0419(7)$ & $-0.0036(7)$ & $-0.0003(7)$ & $-0.0106(6)$ \\
\hline $\mathrm{C}(8)$ & $4 a$ & $0.1827(4)$ & $0.8210(3)$ & $0.75503(9)$ & $0.046(1)$ & $0.044(1)$ & $0.0314(9)$ & $-0.004(1)$ & $0.0005(9)$ & $-0.0082(8)$ \\
\hline
\end{tabular}


Table 3. (Continued)

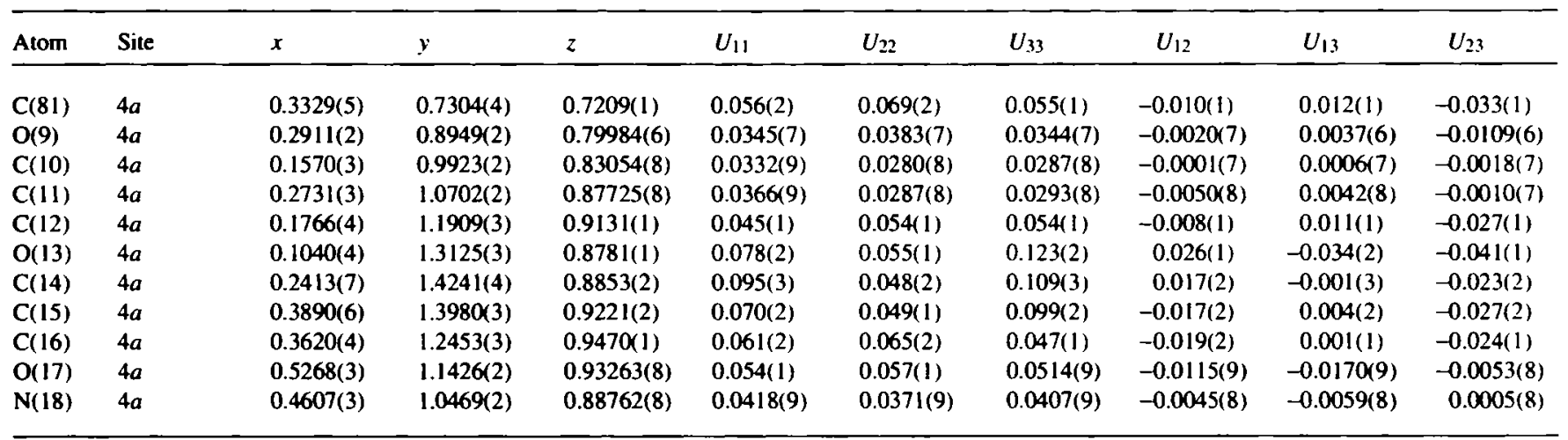

Acknowledgments. We are grateful to the Fonds der Chemischen Industrie, Bayer $A G$ and Hoechst $A G$ for financial support. We also thank Ms. S. Bucovarova for experimental assistance and Dr. Wolfgang Frey for help with the preparation of the files.

\section{References}

I. Leibold, T.: Furan-Nitriloxid-Cycloaddukte - diastereomerenreine Vorläufer für die Synthese von biologisch aktiven Desoxynojirimycin- und Castanospermin-Analoga. Dissertation, Universität Stuttgart, Germany 1995.

2. Müller, I.; Jäger, V.: Synthesis of amino sugars via isoxazolines. Nitrile oxide-furan adducts as key intermediates in the isoxazoline route towards novel amino sugar derivatives. Tetrahedron 41 (1985) 3519-3528.

3. Jäger, V.; Müller, R.; Leibold, T.; Hein, M.; Schwarz, M.; Fengler, M.; Jaroskova, L.; Pätzel, M.; LeRoy, P.-Y.: Synthesis of glycosidase-inhibiting iminopolyols via isoxazolines. Bull. Soc. Chim. Belg. 103 (1994) 491-507.
4. Liu, K.-C.; Shelton, B. R.; Howe, R. K.: A particularly convenient preparation of benzohydroximinoyl chlorides (nitrile oxide precursors). J. Org. Chem. 45 (1980) 3916-3918.

5. Henkel, S.; Leibold, T.; Jäger, V.: Crystal structure of 1,3:2,4-di-O-ethylidene-L-xylose oxime, $\mathrm{C}_{9} \mathrm{H}_{15} \mathrm{NO}_{5}$. Z. Kristallogr. NCS 213 (1998) 65-66.

6. Henkel, S.; Leibold, T.; Jäger, V.: Crystal structure of $(3 \mathrm{a} R, 6 \mathrm{a} R)-3$ [( $1 R, 2 S, 3 S)$-1,3:2,4-di-O-ethylidene-1,2,3,4-tetrahydroxy-1-butyl]-3a,6adihydrofuro $[2,3-d]$ isoxazole, $\mathrm{C}_{13} \mathrm{H}_{17} \mathrm{NO}_{6}$. $\mathrm{Z}$. Kristallogr. NCS 213 (1998) 67-68.

7. Sheldrick, G. M.: Phase Annealing in SHELX-90: Direct Methods for Large Structures. Acta Crystallogr. A46 (1990) 467-473.

8. Sheldrick, G. M.: SHELXL-93. Program for refining crystal structures. University of Göttingen, Germany 1993. 